\title{
A Compact SIW Power Divider for Dual-Band Applications
}

\author{
Rusan Kumar BARIK ${ }^{1}$, Qingsha S. CHENG ${ }^{1}$, Nrusingha Charan PRADHAN ${ }^{2}$, \\ Karthikeyan SHOLAMPETTAI SUBRAMANIAN ${ }^{2}$
}

\footnotetext{
${ }^{1}$ Dept. of Electrical and Electronic Engineering, Southern University of Science and Technology, Shenzhen, China

2 Dept. of Electronics and Communication Engineering, National Institute of Technology, Tiruchirappalli, Tamil Nadu-620015, India
}

rusanbarik@gmail.com, chengqs@ @ustech.edu.cn,nrusinghpradhan01@gmail.com, sskarthikeyan@nitt.edu

Submitted November 7, 2019 / Accepted January 27, 2020

\begin{abstract}
In this paper, a novel design of highly compact power divider employing substrate-integrated waveguide (SIW) is proposed for dual-band applications. The double-ring asymmetric complimentary split-ring resonators (CSRRs) are utilized to obtain dual-band operation. The asymmetric double-ring CSRRs create mixed magnetic and electric coupling resulting two distinct resonating frequencies which exhibits bandpass behaviour below the resonating frequency of the cavity. The resonating passbands can be designed individually by varying the dimensions of the proposed CSRRs. In addition, the position of output ports can be varied to achieve arbitrary power division. To demonstrate the proposed analysis, three prototypes (two equal power division and one unequal power division) of dual-band SIW power dividers are designed and fabricated. Measurement performance provides a good consistency with that of simulated one. The circuit areas of the fabricated prototypes 1,2 and 3 excluding microstrip transitions are $0.053 \lambda_{\mathrm{g}}{ }^{2}$, $0.088 \lambda_{g}{ }^{2}$ and $0.033 \lambda_{g}{ }^{2}$, respectively. The proposed design process exhibits dual-band performance with smaller circuitarea, suitable isolation and hence appropriate for dual-band communication services.
\end{abstract}

\section{Keywords}

Dual-band, SIW, compact, power divider

\section{Introduction}

The Development power dividers (PDs), directional couplers, bandpass filters and diplexers etc, employing SIW technology have gained a great attraction due to their larger Q-factor, lower insertion-loss, low cost, compact size and easily integrable with other components [1-7]. The SIW power divider becomes a key component at millimeter wave band and widely used in array antennas, multiplexers and power amplifiers (PAs). In recent years, the development of SIW power dividers with low insertion loss, broadband, miniaturization has been attracted many researchers [8-22]. A high isolation SIW power divider based on the fixed width lines has been designed [8]. In [9], a miniaturized power divider has been designed using 1/32th mode SIW resonator. In [10], a 3-dB E-plane PD with the use of resistive layer has been implemented applying SIW technology. In [11], a two layer PD employing SIW has been developed to obtained out-of-phase response. A PD has been realized on SIW using EBG (electromagnetic band-gap) structures for broadband and wide out-of-band rejection [12].

Recently, several designs have been presented to improve isolation and to achieve filtering response. A compact power divider employing quarter-mode circular cavity has been designed to obtain filtering response [13]. A compact SIW power divider has been implemented applying defected ground structure (DGS) for improved isolation and bandpass response [14]. The T-junction SIW structure has been employed to design a PD with distinct power distribution [15]. A compact power divider using SIW has been developed to obtain stop-band performance [16]. In [17], A PD employing SIW has been designed by applying CSRR for compactness and filtering response. In [18], half-mode SIW structure has been used to develop a power divider for compactness and distinct power division. An eight-way power divider applying SIW has been presented to achieve low insertion-loss [19]. The Y-junction four-way PD employing SIW has been developed for broadband application [20]. In [21], a multi-layer power divider employing SIW has been implemented to obtain out-of-phase response. In [22], the SIW magic-T has been designed for wideband application.

Miniaturized dual-frequency microwave devices such as PDs, filters and branch-line baluns facilitate the utilization of space and operating at distinct frequency bands simultaneously for modern wireless communication services. Accordingly, they have gained significant interest in the implementation of dual-frequency PD employing SIW. In recent 
years, few development of dual-frequency PD using SIW have been reported [23-24]. In [23], half-mode SIW with slots and resistive elements has been used to develop a PD for dual-frequency communication services. This PD improves isolation by using four resistors but increase the prototype size as well as insertion loss. SIW triangular cavities along with resistive elements have been employed to design dual-frequency power divider in [24]. This prototype improves isolation, occupies larger circuit-area and increases insertion-loss due to the usage two resistors.

This work presents the design of a novel compact SIW power divider for dual-band applications. By loading doublering asymmetric CSRRs on the top conducting surface of the cavity, a dual-band frequency response is obtained. Different center frequencies are achieved by varying the design parameters of the CSRRs. In addition, the position of output ports can be varied to achieve arbitrary power division. For verification of the proposed concept, three SIW power dividers (two equal power division and one unequal power division) working at two distinct resonating frequencies are implemented and fabricated. The measurement performances are in good consistency with that of simulated one. The isolation and return loss at each resonating passband are obtained as $9 \mathrm{~dB}$ and $16 \mathrm{~dB}$, respectively. The phase and magnitude imbalances are well below $0.7 \mathrm{~dB}$ and $1^{\circ}$, respectively, at all the resonating frequencies.

\section{Design Configuration and Analysis}

The schematic configuration of the dual-band power divider (DBPD) employing SIW is depicted in Fig. 1. The proposed topology is consists of a rectangular cavity with linearly arranged metallized vias and CSRRs of unequal dimensions. The double-ring CSRRs are different in size and connected in parallel on the top conducting surface of the cavity. The metallic vias are structured in two parallel rows to obtain electric-walls of the cavity. The direction of the magnetic field is parallel to the metallic-facet of the cavity whereas the electric field is perpendicular to the ground and top conducting surface. The mixed magnetic and electric couplings are created by the split rings and slots, resulting in the resonance frequencies. This resonance frequencies can be produced below the resonating frequency of the cavity. Three design steps are followed to develop the dual-frequency power divider using SIW: firstly, the resonating frequency of the dominant mode $\left(\mathrm{TE}_{10}\right)$ is computed by employing the formulas [2]:

$$
\begin{gathered}
f_{\mathrm{c}\left(\mathrm{TE}_{10}\right)}=\frac{c}{2 W_{\mathrm{s}} \sqrt{\epsilon_{\mathrm{r}}}}, \\
W_{\mathrm{s}}=W-\frac{d^{2}}{0.95 s}
\end{gathered}
$$

where $W_{\mathrm{s}}$ is specified as effective width of the cavity. The diameter and the center-to-center distance of the via-holes are specified by $d$ and $s$, respectively. In order to keep leakage loss as minimum as possible, the diameter and pitch need to be chosen as $d / \lambda_{\mathrm{g}} \leq 0.1$ and $d / s \geq 0.5$ ( $\lambda_{\mathrm{g}}$ is defined as the guided wavelength at the resonating frequency).

Secondly, the resonance generated due to the mixed coupling of magnetic and electric fields of the rectangular CSRRs is employed to achieve the passband frequencies below the resonating frequency of the SIW. The distinct resonating frequencies can be achieved by varying the parameters of the rectangular CSRRs. The variables $L_{1}, L_{4}$ and $W_{1}$ are varied to determine the different operating frequency bands. Finally, the variables of the resonators are optimised to achieve required resonance frequencies.

Initially, a power divider is structured using SIW cavity of dimensions $10.8 \mathrm{~mm} \times 4.8 \mathrm{~mm}$. The diameter and the center-to-center distance of the via-holes are assigned as 0.6 and $1.2 \mathrm{~mm}$, respectively. The simulated magnitude responses and field distribution of the SIW PD without resonators are depicted in Fig. 2. Then, the asymmetric doublering rectangular CSRRs connected in shunt are engraved on the cavity surface to obtain two distinct resonating frequencies. The operating passbands below the cavity resonating frequency are generated by the mixed coupling of magnetic and electric fields of the rectangular CSRRs. By varying the parameters of the resonators, the resonating frequencies of the SIW PD can be tuned as per the requirement.

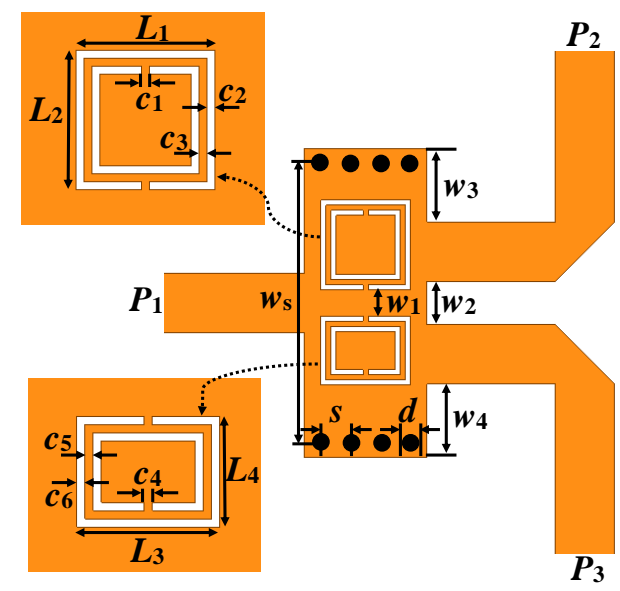

Fig. 1. Schematic configuration of the proposed SIW power divider.

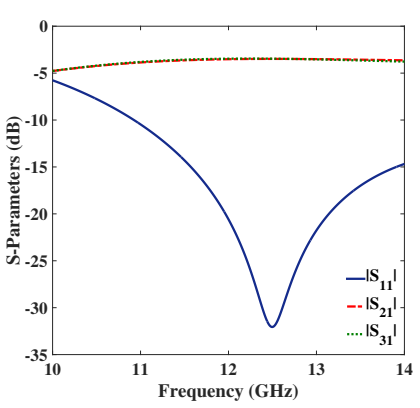

(a)

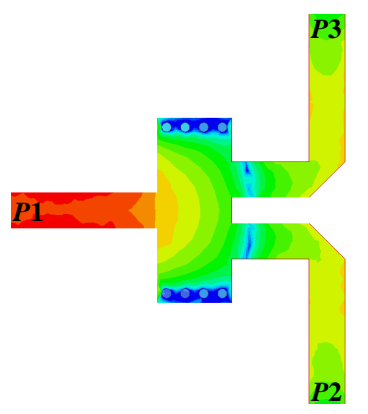

(b)
Fig. 2. Performance of SIW PD without resonators. (a) Magnitude resoponse and (b) E-field distribution. 


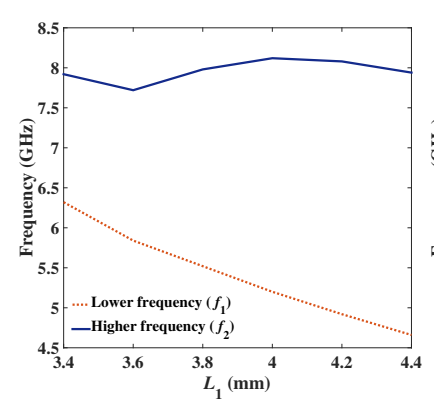

(a)

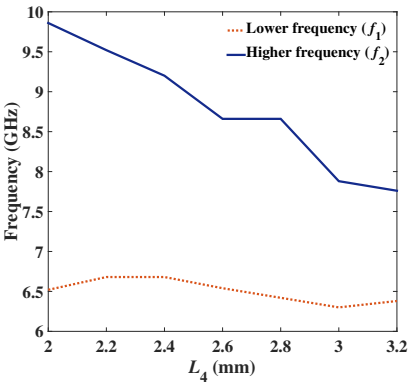

(b)

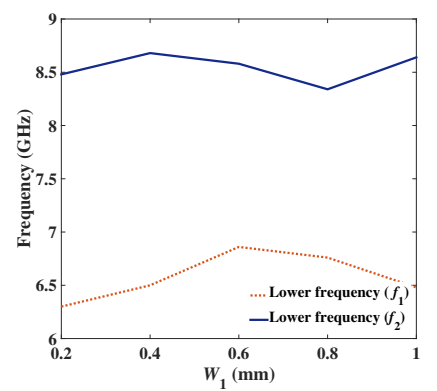

(c)

Fig. 3. Variation of resonant frequencies with (a) $L_{1}(\mathrm{~mm})$, (b) $L_{4}(\mathrm{~mm})$ and (c) $W_{1}(\mathrm{~mm})$.

Figure 3 shows that the resonant frequencies $\left(f_{1}\right.$ and $\left.f_{2}\right)$ can be tuned by $L_{1}, L_{4}$ and $W_{1}$. From Fig. 3(a), it is observed that the lower resonant frequency tuned significantly with respect to $L_{1}$. The higher resonant frequency tuned significantly with $L_{4}$ as depicted in Fig. 3(b). The resonant frequencies can be varied with the space between two resonators $\left(W_{1}\right)$ as illustrated in Fig. 3(c).

\subsection{Implementation of Dual-Band SIW PD with Equal Power Division}

In this section, dual-band SIW power dividers with equal power division are developed and implemented. To achieve dual-band operation, two unequal CSRRs are loaded on the surface of the SIW. Due to mixed magnetic and electric coupling, the passbands are obtained below the waveguide cut-off frequency. The output ports (ports 2 and 3) are placed symmetric with respect to input port (port 1) to achieve equal power division. To demonstrate the analysis, two dual-band SIW power divider with equal power division are developed and fabricated. The prototypes are operating at $6.5 / 8.65 \mathrm{GHz}$ (for prototype 1) and $8.4 / 11.65 \mathrm{GHz}$ (for prototype 2). The SIW DBPDs are fabricated on RT/Duroid 5870 substrate having thickness $=0.787 \mathrm{~mm}, \epsilon_{\mathrm{r}}=2.33$ and $\tan \delta=0.0012$. The optimised dimensions for prototype 1 are: $W_{s}=10.8, W_{1}=0.5, W_{2}=1.69, s=1.2, d=0.6, L_{1}$ $=3.5, L_{2}=3.5, L_{3}=3.5, L_{4}=2.7, c_{1}=0.2, c_{2}=0.2, c_{3}$ $=0.2, c_{4}=0.2, c_{5}=0.2, c_{6}=0.2$ and for prototype 2 are: $W_{s}=10.8, W_{1}=0.5, W_{2}=1.69, s=1.2, d=0.6, L_{1}=2.5$, $L_{2}=2.5, L_{3}=3.5, L_{4}=2.8, c_{1}=0.2, c_{2}=0.21, c_{3}=0.2$, $c_{4}=0.2, c_{5}=0.2, c_{6}=0.23$. Units are in millimeters. The fabricated DBPD prototypes are shown in Fig. 4. The E-field distributions of the proposed DBPDs are illustrated in Figs. 5 and 6 for prototypes 1 and 2, respectively.
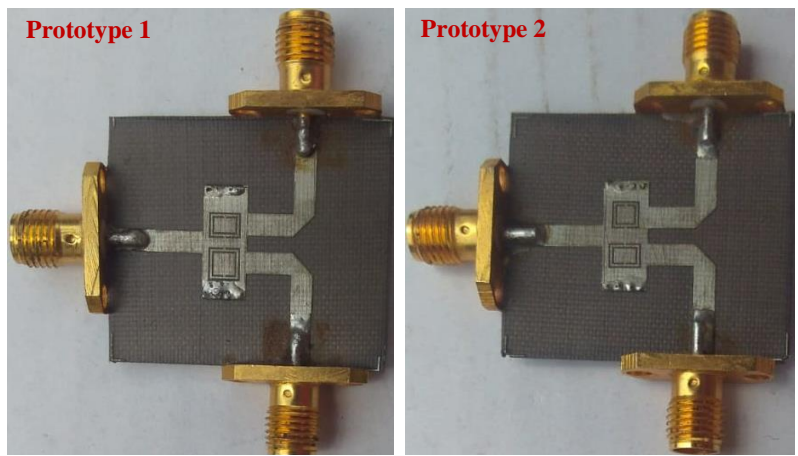

Fig. 4. Fabricated prototypes of proposed DBPD with equal power division.

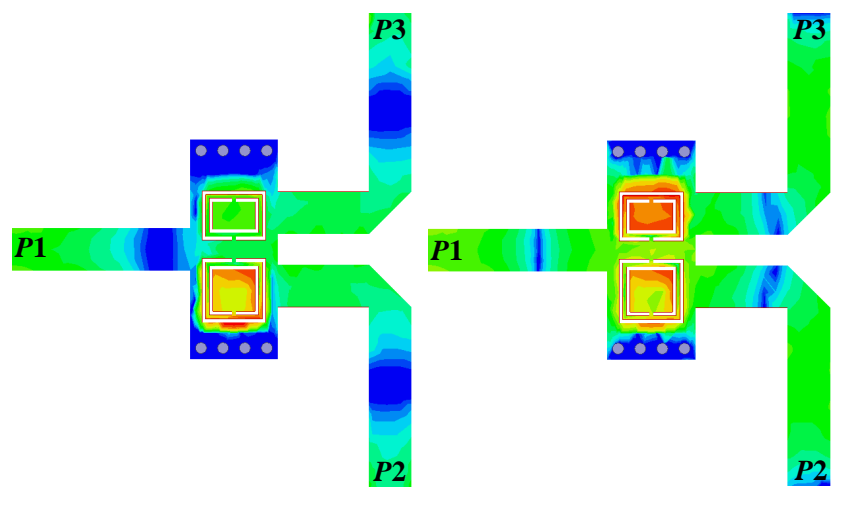

(a)

(b)

Fig. 5. E-field distribution of the DBPD (prototype 1). (a) Lower resonating frequency $(6.5 \mathrm{GHz})$ and (a) higher resonating frequency $(8.65 \mathrm{GHz})$

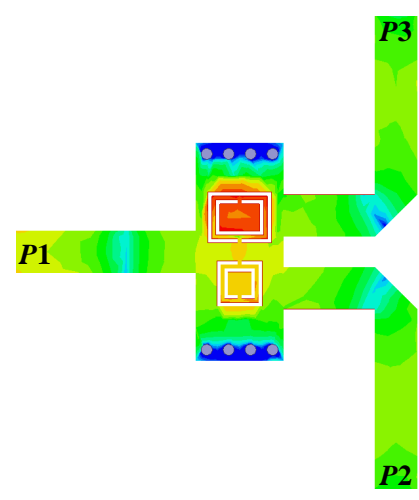

(a)

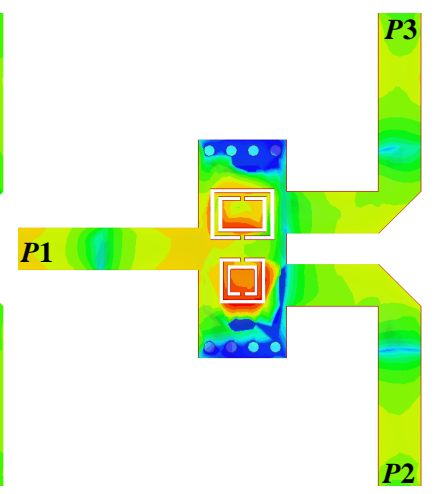

(b)
Fig. 6. E-field distribution of the DBPD (prototype 2). (a) Lower resonating frequency $(8.4 \mathrm{GHz})$ and (a) higher resonating frequency $(11.65 \mathrm{GHz})$.

\subsection{Implementation of Dual-Band SIW PD with Unequal Power Division}

In this section, dual-band SIW power divider with arbitrary power division is developed and implemented. To achieve arbitrary power division, the output ports (port 2 and 3) are placed asymmetric with respect to center of input port 1. Power division between two output ports can be controlled by varying the parameter $W_{2}$. The power division increases by increasing the parameter $W_{2}$. The output port 2 
(port 3) receives more power than output port 3 (port 2) when placed nearer to the center with respect to input port 1 . To validate the analysis, a dual-band SIW power divider with power division of $4 \mathrm{~dB}$ operating at $4.35 \mathrm{GHz}$ and $5.72 \mathrm{GHz}$ is designed and fabricated. In prototype 3 , the output port 2 receives more amount of power than the port 3 . The optimised dimensions of the fabricated prototype 3 are: $W_{\mathrm{s}}=$ $12.4, W_{1}=0.4, W_{2}=4.69, W_{3}=1.595, W_{4}=3.095, s=1.6, d$ $=0.8, L_{1}=4, L_{2}=4, L_{3}=5.5, L_{4}=4, c_{1}=0.2, c_{2}=0.2, c_{3}=$ $0.2, c_{4}=0.2, c_{5}=0.2, c_{6}=0.2$. Units are in millimetres. The fabricated prototype is depicted in Fig. 7. The E-field distribution of the proposed SIW DBPD with power division of $4 \mathrm{~dB}$ is illustrated in Fig. 8. It can be seen that the maximum field is concentrated at the center of the SIW cavity.

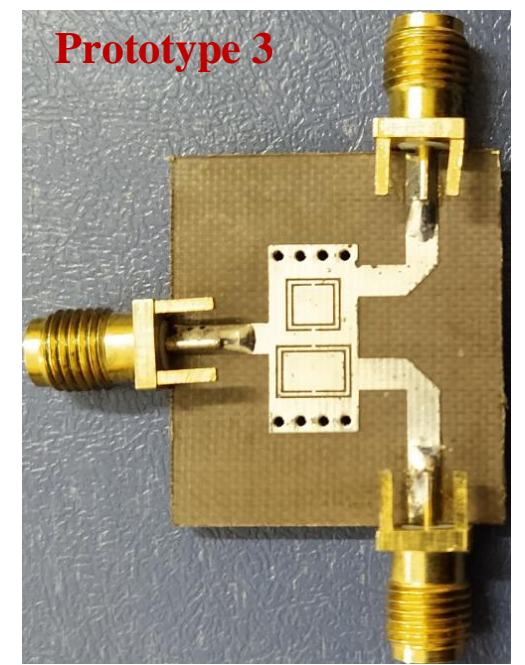

Fig. 7. Fabricated prototypes of proposed DBPD with unequal power division.

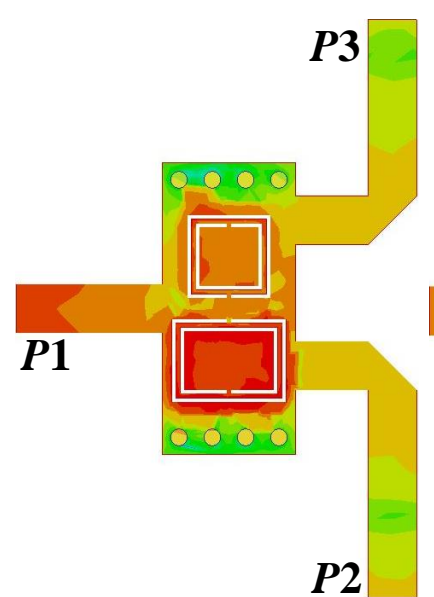

(a)

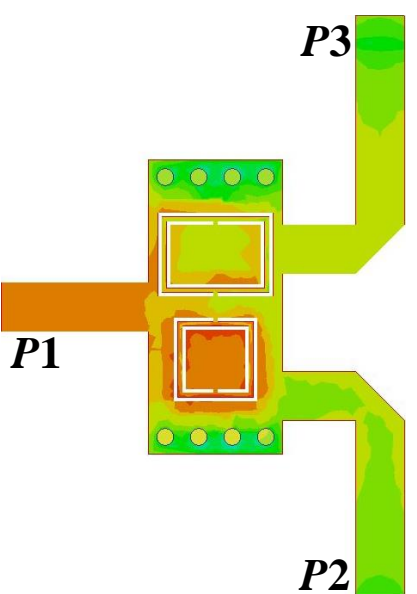

(b)
Fig. 8. E-field distribution of the DBPD (prototype 3). (a) Lower resonating frequency $(4.35 \mathrm{GHz})$ and (a) higher resonating frequency $(5.72 \mathrm{GHz})$.

\section{Performance Analysis of Proposed Dual-Band SIW Power Dividers}

This section demonstrates the comparative analysis of the proposed dual-band SIW PD prototypes. Comparison of performances for all the fabricated prototypes are discussed in detail. To highlight the advantages of the proposed work, the state-of-the-arts SIW power dividers are summarized and discussed. The R\&S ZVL network analyzer is employed to obtain the measurement parameters for all fabricated prototypes. A good consistency is found between the simulated and tested performances.

\subsection{Performances of Dual-Band SIW PDs with Equal Power Division}

Figures 9 and 10 illustrate the simulated and tested amplitude response and isolation of the dual-frequency SIW power dividers, respectively. From the responses, it is seen that two transmission zeros are generated after the passbands resulting good out-of-band suppression. The simulated and tested return loss are greater than $-16 \mathrm{~dB}$ at each frequency band. The tested and simulated performances of the fabricated prototypes are summarized in Tab. 1. The tested and simulated phase differences of the SIW DBPDs are illustrated in Figs. 11 (prototype-1) and 12 (prototype-2). The tested phase differences between output ports (at $f_{1} / f_{2}$ ) of the prototype 1 and 2 are $0.79^{\circ} / 1.45^{\circ}$ and $0.74^{\circ} / 0.61^{\circ}$, respectively.

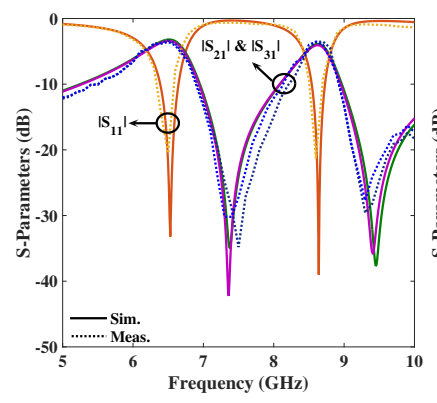

(a)

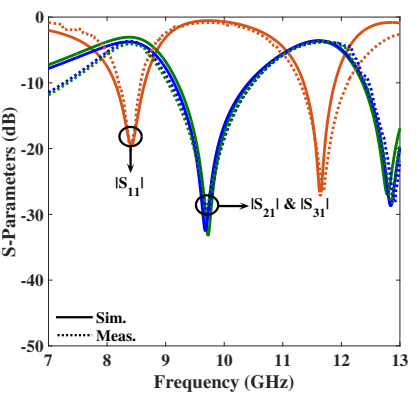

(b)
Fig. 9. Simulated and measured amplitude response of the proposed DBPDs. (a) Prototype-1 and (b) prototype- 2 .

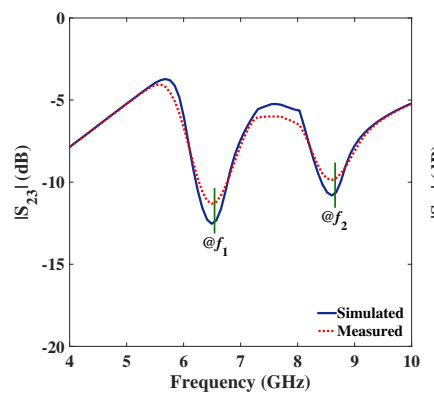

(a)

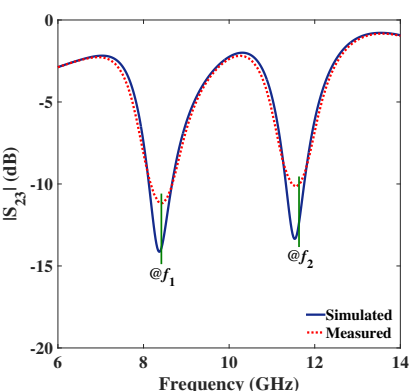

(b)
Fig. 10. Isolation $\left(\left|S_{32}\right|\right)$ of the fabricated DBPDs (a) Prototype-1 and (b) prototype-2. 


\begin{tabular}{|l|l|l|l|l|l|l|}
\hline Designs & Type & $\left|\boldsymbol{S}_{\mathbf{1 1}}\right|[\mathrm{dB}]$ & $\left|\boldsymbol{S}_{\mathbf{2 1}}\right|[\mathrm{dB}]$ & $\left|\boldsymbol{S}_{\mathbf{3 1}}\right|[\mathrm{dB}]$ & $\left|\boldsymbol{S}_{\mathbf{2 3}}\right|[\mathrm{dB}]$ & PD \\
\hline \multirow{2}{*}{ Prototype 1 } & $\begin{array}{l}\text { Simulated } \\
\left(f_{1} / f_{2}\right)\end{array}$ & $-22.46 /-27.56$ & $-3.20 /-3.89$ & $-3.39 /-4.11$ & $12.5 / 10.6$ & $0^{\circ} \pm 2^{\circ}$ \\
\cline { 2 - 6 } & $\begin{array}{l}\text { Measured } \\
f_{1} / f_{2}\end{array}$ & $-20.5 /-16.6$ & $-3.35 /-3.45$ & $-3.56 /-3.77$ & $11.2 / 9.75$ & $0^{\circ} \pm 2^{\circ}$ \\
\hline \multirow{2}{*}{ Prototype 2 } & $\begin{array}{l}\text { Simulated } \\
f_{1} / f_{2}\end{array}$ & $-20.33 /-26.97$ & $-3.06 /-3.67$ & $-3.78 /-3.58$ & $14.0 / 11.87$ & $0^{\circ} \pm 2^{\circ}$ \\
\cline { 2 - 6 } & $\begin{array}{l}\text { Measured } \\
f_{1} / f_{2}\end{array}$ & $-19.37 /-24.41$ & $-3.67 /-3.8$ & $-4.08 /-3.71$ & $11.1 / 9.89$ & $0^{\circ} \pm 2^{\circ}$ \\
\hline
\end{tabular}

Tab. 1. The measured and simulated performances of the dual-frequency SIW PDs with equal power division.

\begin{tabular}{|l|l|l|l|l|l|}
\hline Designs & Type & $\left|\boldsymbol{S}_{\mathbf{1 1}}\right|[\mathrm{dB}]$ & $\boldsymbol{\Delta P}[\mathrm{dB}]$ & $\left|\boldsymbol{S}_{\mathbf{2 3}}\right|[\mathrm{dB}]$ & $\mathbf{P D}$ \\
\hline \multirow{2}{*}{ Prototype 3 } & $\begin{array}{l}\text { Simulated } \\
\left(f_{1} / f_{2}\right)\end{array}$ & $-28.44 /-21.35$ & $4.37 / 4.04$ & $17.82 / 13.59$ & $0^{\circ} \pm 3^{\circ}$ \\
\cline { 2 - 6 } & $\begin{array}{l}\text { Measured } \\
f_{1} / f_{2}\end{array}$ & $-26.1 /-20.68$ & $4.19 / 4.08$ & $15.55 / 16.52$ & $0^{\circ} \pm 3^{\circ}$ \\
\hline
\end{tabular}

Tab. 2. The measured and simulated performances of the dual-band SIW PDs with unequal power division.

\begin{tabular}{|l|l|l|l|l|l|l|}
\hline Ref. & Band & CF $[\mathrm{GHz}]$ & RL $[\mathrm{GHz}]$ & ISL $[\mathrm{GHz}]$ & MI $[\mathrm{GHz}]$ & Size $\left[\mathrm{d}_{\mathrm{g}}{ }^{2}\right]$ \\
\hline$[9]$ & 1 & 2.4 & $>10$ & $>13$ & \pm 0.4 & 0.49 \\
\hline$[10]$ & 1 & 10 & $>10$ & $>15^{*}$ & \pm 1.0 & 3.128 \\
\hline$[12]$ & 1 & 4.5 & 13 & NR & \pm 1.1 & 0.674 \\
\hline$[13]$ & 1 & 9.1 & 16 & NR & \pm 0.6 & Not Given \\
\hline$[14]$ & 1 & 9 & 12 & $>20^{*}$ & \pm 1.1 & 1.09 \\
\hline$[15]$ & 1 & 6 & $>15$ & NR & \pm 0.5 & Not Given \\
\hline$[16]$ & 1 & 4.3 & $>10.6$ & NR & \pm 1.2 & 0.645 \\
\hline$[17]$ & 1 & 1.54 & $>13$ & NR & \pm 1.0 & 0.011 \\
\hline$[18]$ & 1 & 9.77 & $>12.5$ & $>10^{*}$ & \pm 0.3 & 1.175 \\
\hline$[23]$ & $1 / 2$ & $2.3 / 3.5$ & $>16$ & $>16^{*}$ & $\pm 1.8 / \pm 1.4$ & 2.4 \\
\hline$[24]$ & 2 & $5.5 / 8.3$ & $>13$ & $>12^{*}$ & $\pm 0.9 / \pm 1.5$ & 1.913 \\
\hline Prototype $1(\Delta \mathrm{P}=0 \mathrm{~dB})$ & 2 & $6.5 / 8.65$ & $>16$ & $>9$ & $\pm 0.56 / \pm 0.77$ & 0.053 \\
\hline Prototype 2 $(\Delta \mathrm{P}=0 \mathrm{~dB})$ & 2 & $8.4 / 11.65$ & $>19$ & $>9$ & $\pm 0.67 / \pm 0.71$ & 0.088 \\
\hline Prototype 3 $(\Delta \mathrm{P}=4 \mathrm{~dB})$ & 2 & $4.35 / 5.72$ & $>20$ & $>15$ & $\pm 0.25 / \pm 0.17$ & 0.033 \\
\hline
\end{tabular}

Tab. 3. The state-of-the art comparison of the SIW power dividers. (RL: Return Loss, MI: Magnitude Imbalance, CF: Center frequency, ISL: Isolation, NR: Not reported.)
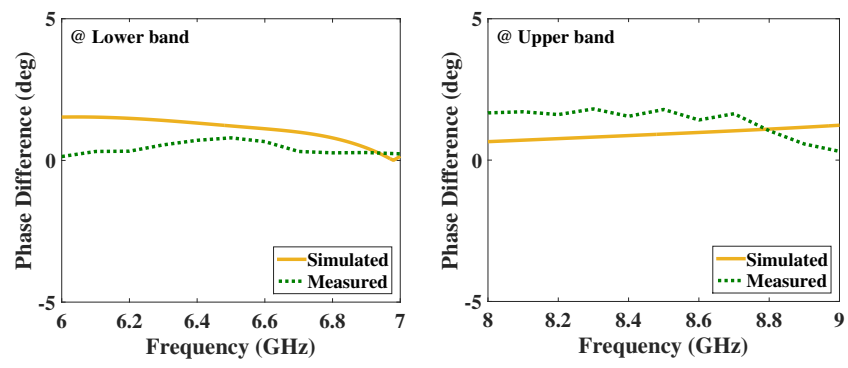

Fig. 11. Phase performances of the fabricated prototype 1.
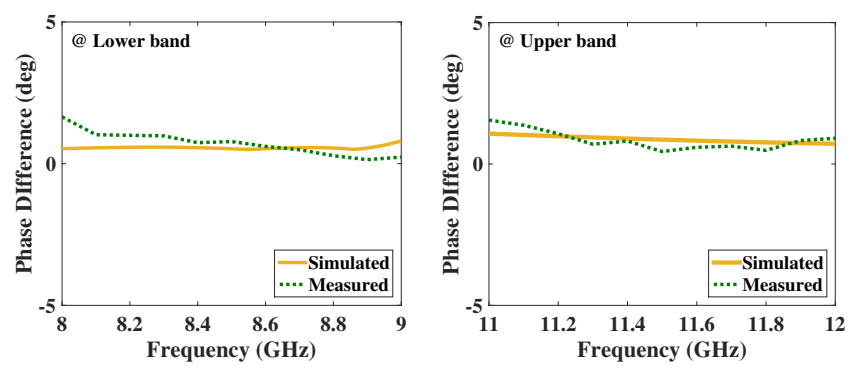

Fig. 12. Phase performances of the fabricated prototype 2.

\subsection{Performances of Dual-Band SIW PD with Unequal Power Division}

The simulated and tested magnitude responses and isolation of the dual-frequency SIW PD with power division $(\Delta P)$ of $4 \mathrm{~dB}$ are illustrated in Figs. 13 and 14, respectively. From the plot, it is noticed that the tested reflection coefficient and power division at $4.35 \mathrm{GHz}$ and $5.72 \mathrm{GHz}$ are better than $-20 \mathrm{~dB}$ and $4 \mathrm{~dB}$, respectively. The measured isolations at $4.35 \mathrm{GHz}$ and $5.72 \mathrm{GHz}$ are greater than $15 \mathrm{~dB}$. The tested and simulated performances of the fabricated prototype 3 is summarized in Tab. 2. The tested and simulated phase differences of the SIW DBPDs with power division of $4 \mathrm{~dB}$ is illustrated in Fig. 15.

The state-of-the-arts SIW power dividers are compared and illustrated in Tab. 3. It is seen that the SIW power dividers in $[9,10,23,24]$ provide slightly better isolation than the proposed prototypes due to the use of resistive elements in their design. The present prototypes achieve compact size and less insertion loss than reported SIW PDs [9-24]. Therefore the proposed SIW DBPD exhibits the advantages of smaller circuit-area, low insertion-loss and acceptable isolation beneficial for dual-band applications. 


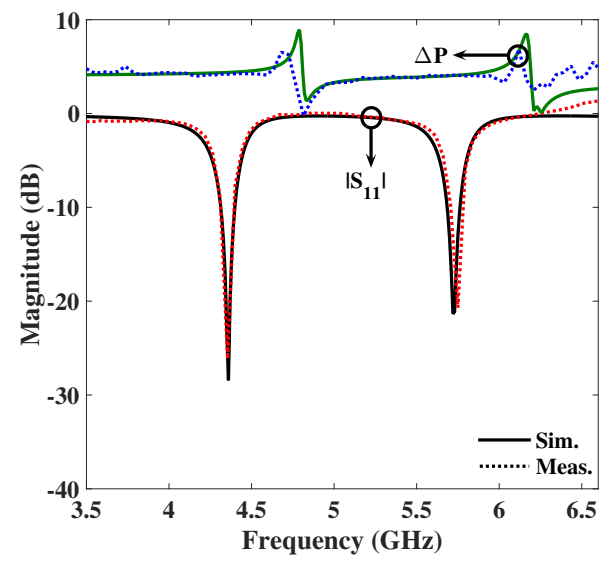

Fig. 13. Simulated and measured amplitude response of the proposed DBPDs (prototype 3) with $\Delta P=4 \mathrm{~dB}$.

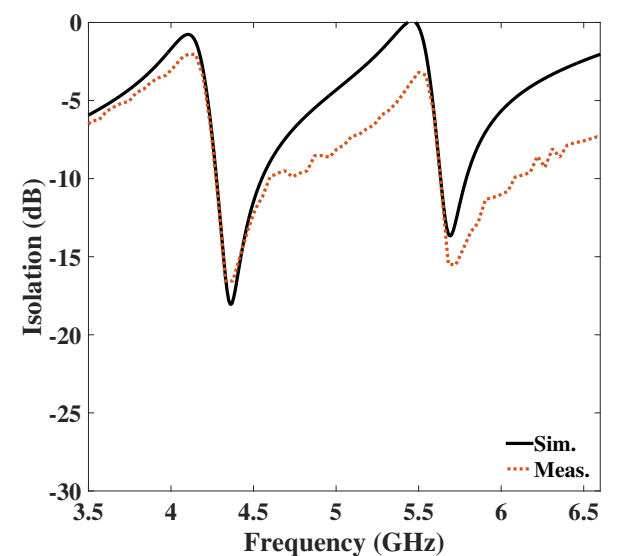

Fig. 14. Isolation $\left(\left|S_{32}\right|\right)$ of the fabricated DBPDs (Prototype-3).
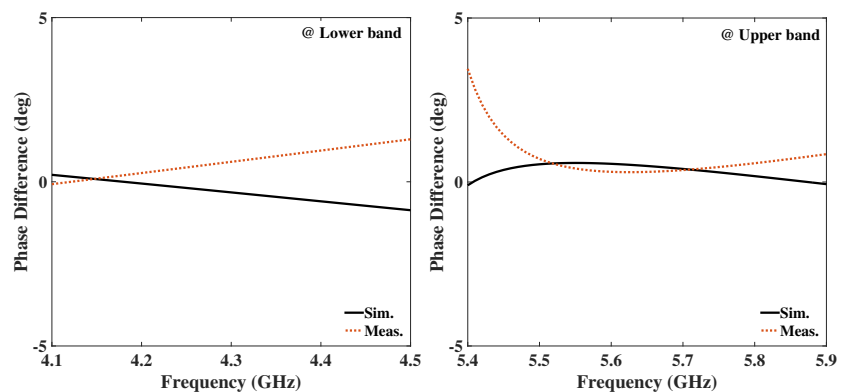

Fig. 15. Phase performances of the fabricated prototype 3 .

\section{Conclusions}

A novel design process for compact SIW power divider has been proposed and implemented for dual-band application. By configuring CSRRs properly on the top of the SIW without increasing in the circuit size, dual-band characteristic has been excited below the waveguide cut-off frequency. Also, varying passbands have been achieved by changing the dimensions of the CSRRs. A parametric study has been presented with a clearer explanation to obtain dual-band operation. A number of cases to tune the resonant frequency have been illustrated. Finally, three prototypes (two equal power division and one unequal power division) of dual-frequency
SIW PD have been fabricated and demonstrated. The measurement performances are in good consistency with that of simulated one, demonstrating that the reported dual-band power dividers exhibit compact size, attractive performance and easy integration at low cost, hence suitable for dual-band wireless communication services.

\section{Acknowledgments}

This work is partially supported by the National Natural Science Foundation of China Grant 61471258 and by Science \& Technology Innovation Committee of Shenzhen Municipality Grant KQJSCX20170328153625183.

\section{References}

[1] DONG, Y., YANG, T., ITOH, T. Substrate integrated waveguide loaded by complementary split-ring resonators and its applications to miniaturized waveguide filters. IEEE Transactions on Microwave Theory and Techniques, 2009, vol. 57, no. 9, p. 2211-2223. DOI: $10.1109 / \mathrm{tmtt} .2009 .2027156$

[2] MXIMO-GUTIERREZ, C., HINOJOSA, J., ALVAREZ-MELCON, A. Design of wide band-pass substrate integrated waveguide (SIW) filters based on stepped impedances. AEU - International Journal of Electronics and Communications, 2018, vol. 100, p. 1-8. DOI: 10.1016/j.aeue.2018.12.022

[3] AGHAYARI, H., NOURINIA, J., GHOBADI, C. Incorporated substrate integrated waveguide filters in propagative and evanescent mode: Realization and comparison. AEU - International Journal of Electronics and Communications, 2018, vol. 91, p. 150-159. DOI: $10.1016 /$ j.aeue.2018.05.008

[4] ZHOU, K., ZHOU, C., WU, W. Substrate integrated waveguide dual-band filter with wide-stopband performance. Electronics Letters, 2017, vol. 53, no. 16, p. 1121-1123. DOI: 10.1049/el.2017.1556

[5] ZHANG, H., KANG, W., WU, W. Dual-band substrate integrated waveguide bandpass filter utilising complementary split-ring resonators. Electronics Letters, 2018, vol. 54, no. 2, p. 85-87. DOI: $10.1049 / \mathrm{el} .2017 .3478$

[6] DONG, Y., WU, C. M., ITOH, T. Miniaturised multi-band substrate integrated waveguide filters using complementary split-ring resonators. IET Microwaves, Antennas \& Propagation, 2012, vol. 6, no. 6, p. 611-620. DOI: 10.1049/iet-map.2011.0448

[7] DONG, Y., ITOH, T. Miniaturized dual-band substrate integrated waveguide filters using complementary split-ring resonators. In Proceedings of the IEEE MTT-S International Microwave Symposium. Baltimore (USA), 2011, p. 1-4. DOI: 10.1109/mwsym.2011.5973189

[8] MOULAY, A., DJERA, T. Wilkinson power divider with fixed width substrate integrated waveguide line and a distributed isolation resistance. IEEE Microwave and Wireless Components Letters, 2018, vol. 28, no. 2, p. 114-116. DOI: 10.1109/lmwc.2018.2790706

[9] KHAN, A. A., MANDAL, M. K. Miniaturized substrate integrated waveguide (SIW) power dividers. IEEE Microwave and Wireless Components Letters, 2016, vol. 26, no. 11, p. 888-890. DOI: $10.1109 /$ lmwc.2016.2615005

[10] PASIAN, M., SILVESTRI, L., RAVE, C., et al. Substrate-integratedwaveguide E-plane 3-dB power-divider/combiner based on resistive layers. IEEE Transactions on Microwave Theory and Techniques, 2017, vol. 65, no. 5, p. 1498-1510. DOI: 10.1109/tmtt.2016.2642938 
[11] CHEN, Q. , XU, J. Out-of-phase power divider based on two-layer SIW. Electronics Letters, 2014, vol. 50, no. 14, p. 1005-1007. DOI: 10.1049/el.2014.0406

[12] CHEN, S. Y., ZHANG, D. S., YU, Y. T. Wideband SIW power divider with improved out-of-band rejection. Electronics Letters, 2013, vol. 49, no. 15, p. 943-944. DOI: 10.1049/el.2013.0979

[13] WANG, X., ZHU, X. Quarter-mode circular cavity substrate integrated waveguide filtering power divider with via-holes perturbation. Electronics Letters, 2017, vol. 53, no. 12, p. 791-793. DOI: $10.1049 / \mathrm{el} .2017 .0697$

[14] HE, Z., JIANG, Y.C., LENG, S., et al. Compact power divider with improved isolation and bandpass response. Microwave and Optical Technology Letters, 2017, vol. 59, no. 7, p. 1776-1781. DOI: 10.1002/mop.30621

[15] LI, T., DOU, W. Broadband substrate-integrated waveguide T-junction with arbitrary power-dividing ratio. Electronics Letters, 2015, vol. 51, no. 3. p. 259-260. DOI: 10.1049/el.2014.3928

[16] CHEN, S., SU, C., YU, Y., et al. A compact two-way equal power divider with enhanced out-of-band rejection based on SIW technology. Microwave and Optical Technology Letters, 2013, vol. 55, no. 7, p. $1638-1640$. DOI: $10.1002 / \mathrm{mop} .27641$

[17] CHOUDHARY, D. K., CHAUDHARY, R. K. A compact SIW based filtering power divider with improved selectivity using CSRR. In Progress in Electromagnetics Research Symposium Fall (PIERS - FALL). Singapore (Singapore), 2017, p. 1334-1337. DOI: $10.1109 /$ piers-fall.2017.8293337

[18] MOZNEBI, A. R., AFROOZ, K. Compact power divider based on half mode substrate integrated waveguide (HMSIW) with arbitrary power dividing ratio. International Journal of Microwave and Wireless Technologies, 2017, vol. 9, no. 3, p. 515-521. DOI: $10.1017 / \mathrm{s} 1759078716000544$

[19] SONG, K., FAN, Y., ZHANG, Y. Eight-way substrate integrated waveguide power divider with low insertion loss. IEEE Transactions on Microwave Theory and Techniques, 2008, vol. 56, no. 6, p. 1473-1477. DOI: $10.1109 /$ tmtt.2008.923897

[20] ZOU, X., TONG, C., YU, D. Y-junction power divider based on substrate integrated waveguide. Electronics Letters, 2011, vol. 47, no. 25, p. 1375-1376. DOI: 10.1049/el.2011.2953

[21] EOM, D., BYUN, J., LEE, H. Multilayer substrate integrated waveguide four-way out-of-phase power divider. IEEE Transactions on Microwave Theory and Techniques, 2009, vol. 57 no. 12, p. 3469-3476. DOI: 10.1109/tmtt.2009.2034311

[22] ZHU, F., HONG, W., CHEN, J., et al. Design and implementation of a broadband substrate integrated waveguide magic-T. IEEE Microwave and Wireless Components Letters, 2012, vol. 22, no. 12, p. 630-632. DOI: 10.1109/1mwc.2012.2226936

[23] SONG, K., ZHU, Y., ZHANG, F. Single- and dual-band filteringresponse power dividers embedded SIW filter with improved output isolation. Scientific Reports, 2017, vol. 7, no. 3361, p. 1529-1533. DOI: $10.1038 / \mathrm{s} 41598-017-03312-9$

[24] WANG, Y., ZHOU, C., ZHOU, K., WU, K. Compact dual-band filtering power divider based on SIW triangular cavities. Electronics Letters, 2018, vol. 54, no. 2, p. 1072-1074. DOI: 10.1049/el.2018.5611

\section{About the Authors...}

Rusan Kumar BARIK received the B.Tech degree in Electronic \& Communication Engineering from Biju Patnaik University of Technology, Rourkela, India in 2012, M.Tech degree in Communication Systems Design and Ph.D. degree in
Electronics Engineering from Indian Institute of Information Technology, Chennai, Tamil Nadu, India in 2015 and 2018, respectively. He joined the Department of Electronic \& Communication Engineering, Christ University Bangalore, India, as an assistant professor in 2018. He is currently a PostDoctoral Researcher with the Southern University of Science and Technology, Shenzhen, China. His research interests include multiband microwave passive devices, multiband antennas, and SIW components.

Qingsha S. CHENG received the B.Eng. and M.Eng. degrees from Chongqing University, Chongqing, China, in 1995 and 1998, respectively, and the Ph.D. degree from McMaster University, Hamilton, ON, Canada, in 2004. In 1998, he joined the Department of Computer Science and Technology, Peking University, Beijing, China. In 1999, he joined the Department of Electrical and Computer Engineering, McMaster University, where he worked as a postdoctoral fellow, a research associate, and a research engineer. He is currently an Assistant Professor with the Department of Electrical and Electric Engineering, Southern University of Science and Technology, Shenzhen, China. His research interests include surrogate modeling, $\mathrm{CAD}$, modeling of microwave circuits, software design technology, and methodologies for microwave CAD.

Nrusingha Charan PRADHAN received the B.Tech. degree from Biju Patnaik University of Technology, India, in 2013, and the M.Tech degree in Electronics Engineering from Pondicherry Central University, Puducherry, India, in 2016. He was with the Integrated Test Range, Defence Research and Development Organisation, Chandipur, India (DRDO-ITR) for a period of two year. He is currently a Research Scholar with the Department of Electronics and Communication Engineering, National Institute of Technology, Tiruchirappalli, India. He has 3 technical papers. His current research interests include microwave integrated circuits, Substrate Integrated Wave Guide Components for Millimeter Wave Applications. Mr. Pradhan is a Student Member of the IEEE AP Society, the MTT Society.

Karthikeyan SHOLAMPETTAI SUBRAMANIAN received the Ph.D. degree from IIT Guwahati, India, in 2011. He has 13 years of educational activity and research experience in the area of RF and microwave. He was the Short-Term academic foreign visit U.K and France. He is currently Assistant Professor with the Department of Electronics and Communication Engineering, National Institute of Technology, Tiruchirappalli, India. He has authored or co-authored more than 100 scientific research papers and technical reports. His current research interests include microwave integrated circuits, biological effects of microwaves, computer-aided design of MICS, metamaterials/frequency selective surfaces (FSSs), fractal antennas, MIC antennas, metamaterial antennas, and substrate-integrated waveguides. Dr. Karthikeyan is a Chair of the IEEE-APS Society Madras chapter. He is a member of the IEEE, IEEE MTT-S, and IEEE AP-S. He is a Life Member of the ISTE. 